\title{
Design of Intelligent Control System for Greenhouse Water Pump Based on Internet of Things
}

\author{
Dingzhu Xue \\ JiLin Agricultural University, ChangChun, 130118, China;9950502@qq.com
}

Keywords: Internet of things; Automatic control; Water Pump

\begin{abstract}
With the popularity of networking technology, agriculture will gradually shift from a manpower centered, depending on the isolated machinery production mode to the information and software centric mode of production, therefore a variety of automatic and intelligent remote control equipment is used extensively. The temperature sensor, humidity sensor, $\mathrm{PH}$ sensor, photometric sensor and CO2 sensor which use the International System in an intelligent water pump control system for a greenhouse can detect the physics parameters of temperature, relative humidity, $\mathrm{PH}$ value, strength of illumination net, soil nutrient and CO2 concentration. By displaying in real-time through a variety of instruments, or as a factor of an auto control pump system used in the automatic control of water pump, the system can ensure a good environment for crops. This system is mainly composed of MCU module for data transmission to control the running state of water pump. Moreover, the system equipped with a remote control device can realize manual remote control. Even manually controlling the water pump can improve the reliability and safety of the pump system in other emergency situations.
\end{abstract}

\section{Introduction}

At present, modern greenhouses management has gradually tended to be more intelligent and automatic, so how to improve labor efficiency become the main problem people focus on. The traditional manual control cannot meet the requirement of people【1】. In addition, people's struggling between the pump and the switch back and forth cost much of the time and laborites. So a remote control device is badly in need of to solve this problem. At the same time, with the development of intelligent control technology, the construction of automatic and intelligent greenhouses system becomes possible 【2-5】. The pump automatic control system with single chip microcomputer interface introduced in this paper can be very convenient to coordinate the rest of the single chip microcomputer system sensor detection equipment to complete the control of the indoor temperature and humidity in the greenhouse.

\section{System Structure}

The irrigation remote control system consists of a single chip microprocessor (data processing, analysis center) and remote control device. The manual operation of the pump can be realized by remote control device, and the automatic operation of the pump can be made by the instruction from the data processing center of the single chip. The operating principle diagram of the automatic pump control system is shown in figure 1.

(1)The design of the system hardware adopts the current popular modular. Hardware system consists of three functional modules, that is, reception, lack of soil environmental temperature and humidity detection module, the water level detection module, single-chip microcomputer control module. The overall system employs custom communication protocols. Considering the characteristics of agricultural application, the control equipment is self-developed not using expensive programmable controller (PLC) and ready-made wireless modem (radio modem) and so on. Therefore, this equipment with the lowest cost in the domestic similar system effectively reduces the cost of the system. Furthermore, it's more convenient for agricultural extension and application【6】. 


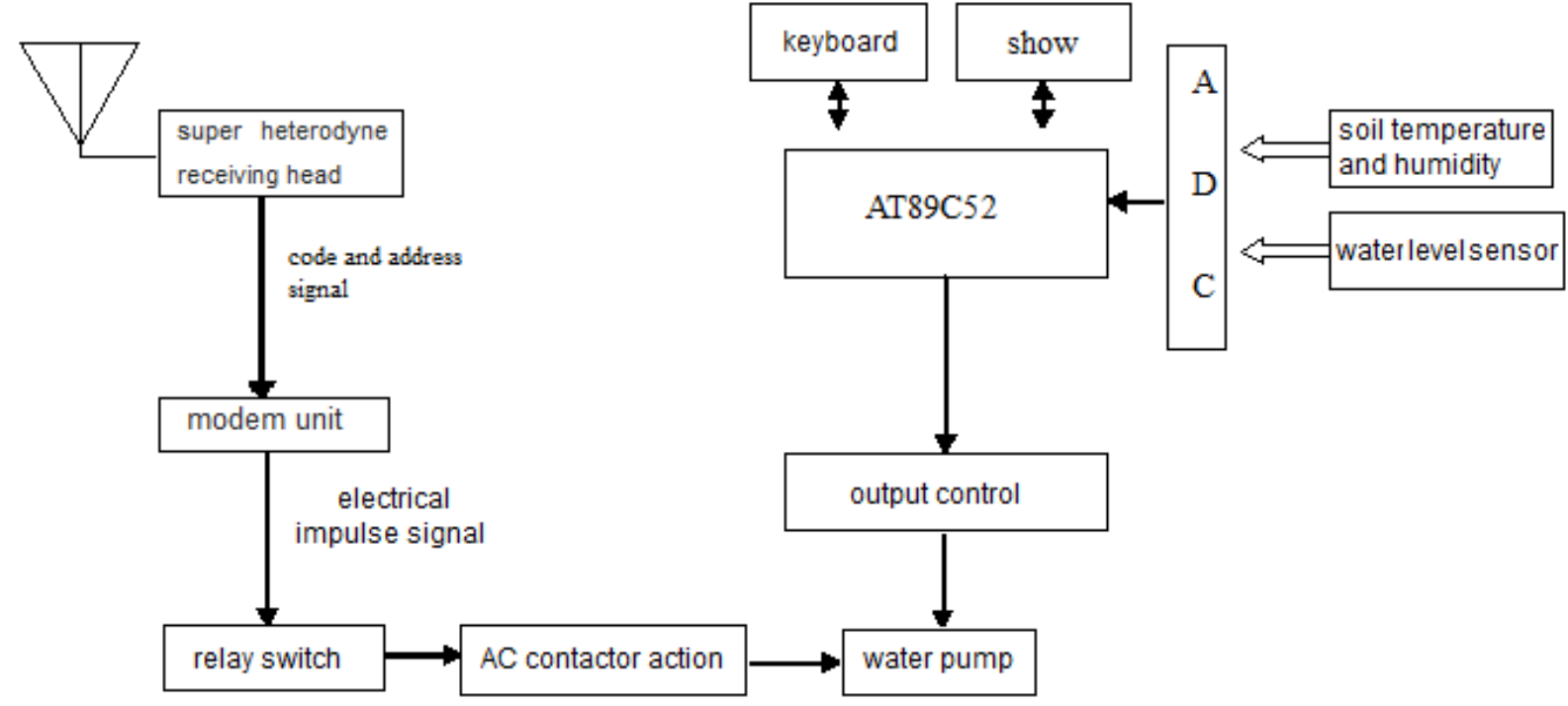

Figure1 The Working Principle of Automatic Pump Control System

(2) Wireless transmission scheme uses PT2262 / PT2272 coding decoding circuit, 315 MHZ transmitting frequency and $8 \mathrm{~km}$ Maximum control radius, 531441 address code is available in switch, which can effectively avoid Interference frequency and interference signal. Wireless receiving head adopts $315 \mathrm{MHZ}$ super heterodyne to receive a receiving circuit formed by MICRF211 chip. The temperature range is from $-30^{\circ} \mathrm{C}$ to $85^{\circ} \mathrm{C}$, which can be adapted to the high temperature environment of greenhouse 【7】 .

(3) A large current mutation will be formed when the Irrigation water pump is operated, so electromagnetic interference is very serious. If certain technical measures cannot be adopted, the system cannot work properly. Staggered water pump switch, data sampling, data transmission time, setting the watchdog circuit, photoelectric isolation, increasing the bus drive capacity and other hardware measures can ensure the stability of the system 【7-8】.

(4) There is pump phase protection circuit in the design of the system. Once the circuit becomes abnormal, the pump can be stopped automatically. Thus the normal operation of the pump can be ensured and the service life can be extended which make the circuit work smoothly 【9】 .

(5) The system controls the best environmental parameters of crop growth in different periods through single chip microcomputer to realize the intelligent management of the greenhouse【10】 . It's characterized with easy operation, high automatization and good human-computer interaction, which can improve the quality and efficiency of production. Data processing center controls the running status of the pump in real time data communication. So the temperature and humidity for the greenhouses can be controlled in the scientific and ideal environment suitable for the growth of the plants.

\section{The Design of Hard Ware}

The hardware is composed of manual remote control and MCU with sensor intelligent control system. The real-time monitoring network of greenhouse centers on single-chip microprocessor. Other components such as soil temperature and humidity sensors, air temperature and humidity sensor, liquid level sensor, wireless transceiver module, liquid crystal display module work together.

The classic design of the current circuit is used in most of the system. The remote control circuit, a low power and low cost universal coding and decoding circuit composed of PT2262/2272 transceiver circuit is made by CMOS technology produced by PuCheng company in TaiWan.

In this system, 315M / 433M super heterodyne receiver head module is selected for the remote transmission of the signal. The receiver sensitivity can reach $-114 \mathrm{dBm}$, operating frequency is 315 
$\mathrm{MHz}$ and 433.92MHz. Power supply voltage input range can be widely selected, any value of the voltage between $3 \mathrm{~V}$ and $5.5 \mathrm{~V}$ can be chosen and the power consumption (5.0V/9.6 mA) is low. The continuous data rate of receiving encoded is up to $2.4 \mathrm{KBPS}$ (Manchester code). This module is featured with good selectivity and spurious radiation suppression ability. [14]The temperature range is from $-30^{\circ} \mathrm{C}$ to $85^{\circ} \mathrm{C}$ which can meet the requirement of the high temperature environment of greenhouse.

Microprocessor is a central part of the whole system and single chip is the core of microprocessor. Microcontroller external crystals and reset circuit, is mainly used for soil temperature and humidity sensor, the water pressure sensor data analysis process and the relay control, water pump, such as functional modules. Temperature and humidity sensor DHT11 contains already calibrated digital composite signal output of the temperature and humidity sensor. The measurement range of relative humidity is $20 \% \sim 90 \% \mathrm{RH}$ and celsius temperature is $0 \sim 50^{\circ} \mathrm{C}$. AT35-3 type liquid level sensor produced by Bi He Company in Shenzhen is chosen as pressure type water level sensor. It can be used to measure the opening level of containers and canals, lakes and Wells. It has the characteristics of simple structure, accurate measurement and convenient installation.

Three Lack of Phase Protection of the Water Pump. This design focuses on the lack of phase protection of the pump. When the motor is running, the power missing, the motor still rotating, but it is obvious in low speed. If heating up sharply, the motor may be burned. If the motor is in the state of stopping, once started in the state of lack of phase, the motor buzzing, not turning up. If not cutting off the power for a long time, the motor will be burned. If it is burned because of lack of phase, the motor will be found two sets of winding are black. It indicates that the breakdown, inter-turn short circuit, or phase short circuit occurs. The performance of the protectors must have wide capacity and wide range of applications, so to achieve a wide range of linear current sampling is one of the key technologies of the system. It is difficult for commercial current transformer to achieve wide range linear current sampling due to magnetic saturation. So this paper uses a self-made current transformer to overcome the magnetic saturation, in order to achieve a wide range of current linear sampling.

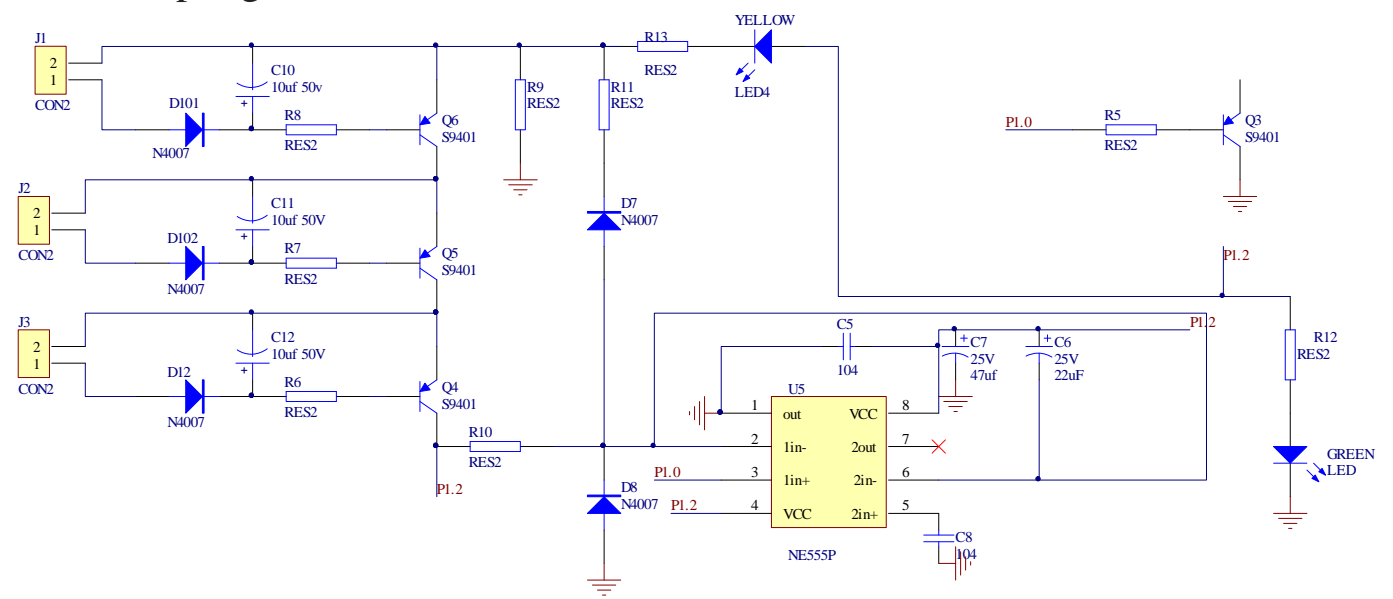

Figure 2 Lack of a protection circuit Schematic diagram

The circuit Schematic diagram of the device is shown in figure 2. J1, J2 and J3 are three inductance coils which are respectively sheathed in the three-phase electric current, which make up the induction electromotive force ring. When the three-phase power lines are in good condition, not producing self-inductance electromotive force, then the circuit will maintain the original state-not working. When there is no current in one of the three phases or when the current fluctuation is large, it will induce the electromotive force in two of the induction coils J1, J2, J3, rectify the diode, charge the capacitor $\mathrm{C} 1$, and add the voltage to the transistor Q4- Q6 transistor conduction. At this time the voltage clutter signal through the resistor R10 is added to the 555 timer trigger side, 3 feet will output a high level so Q3 is conducted, and the relay jump off the main circuit power supply, which can play a role of protecting motor. When the voltage of the 6 pin is $<2 / 3 \mathrm{VCC}$, the voltage of the 3 foot is turned from high level to low level, the Q3 cut-off relay is not working, and the main 
circuit is normally operated. The motor $\mathrm{M}$ is protected. $12 \mathrm{~V} \mathrm{DC}$ power supply is set in the circuit to make the circuit work smoothly.

\section{The Software System Design}

The software is written in C language and microprocessor editor Keil software and adopts modular programming method at the meantime. The program flow diagram is shown in Figure 3. Each part is written separately. Then these parts are connected to the Display function. When the water pump control system works, the main function is used to control the various parts of the Display function.

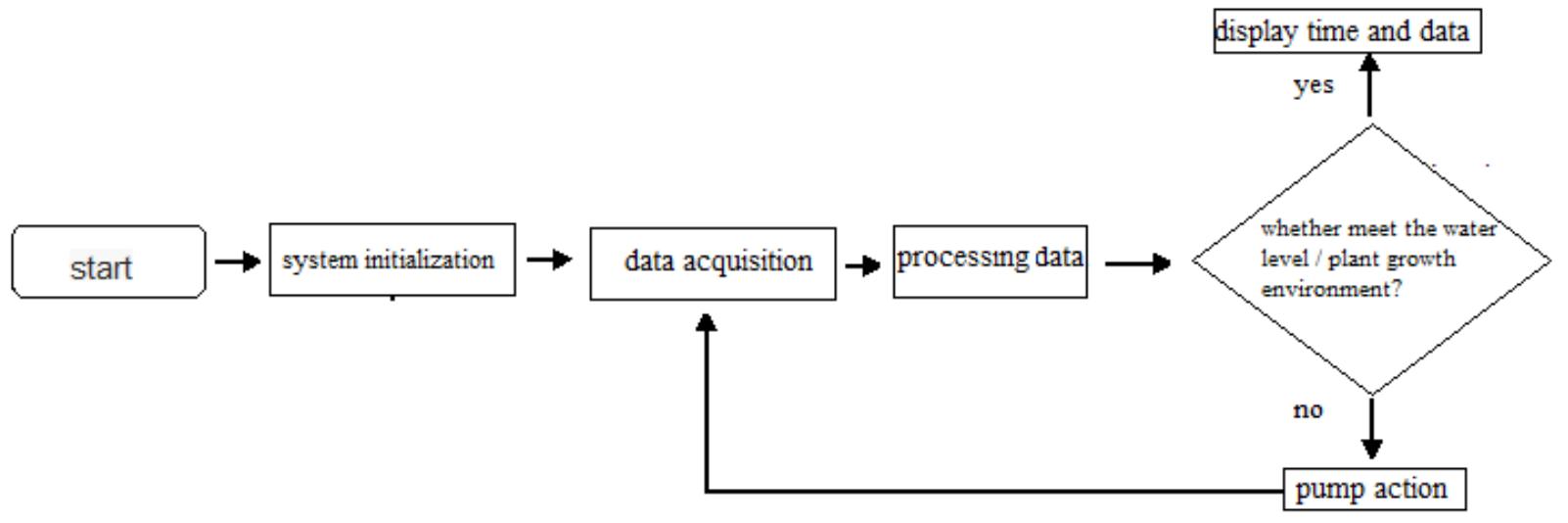

Figure 3 Software flow chart

\section{Summary}

In this paper, the remote management system is designed based on the Internet of things technology. It is characterized by low cost, easy to use and high reliability, which can achieve a number of greenhouses water supply. The new design of the water pump power failure protection basically solves the problem that the motor is still rotating when the power supply is out of phase, causing the motor to rise rapidly and burn the motor.

\section{Acknowledgments}

Funds for the research was provided by scientific research fund of Jilin Agricultural University(No.201620),Jilin province university undergraduate science and technology innovation fund-portable soil comprehensive indicator tester; spark plan Ministry of science and technology (2015GA660004).

\section{References}

[1] Ke Xiaogan. Water level acquisition and remote transmission system research [D]. Nanjing University of Hehai, master's degree thesis, 2013.36-45.

[2] Liu Jingzhi. Foreign mechanical and electrical development of the cause of irrigation and drainage [J]. Netease water conservancy, 2016, 8, 26.

[3] Chen Peiguo. DW15 series universal circuit breaker update and improvement [J]. Electric World, 2005, 8.

[4] Gu Yi, He Xing, Zhang Weidong. Remote monitoring of unattended pumping stations in urban areas [J]. China Water Supply and Drainage, 2014, 20 (9): 84-86.

[5] Guiyun Tian. Principle and Application of Single Chip Microcomputer [M]. Beijing: Higher Education Press, $50 \sim 90$.

[6] Wang Xiaoming. Motor single-chip control [J]. Academic Journal, 2012,13 (15): 1322-1755. 
[7] Huang Xianwu. Sensor principle and application [M] 1st edition, Chengdu, University of Electronic Science and Technology Press, 2005.

[8] Liang Ruilin. Sensor application circuit and the actual production [M] Technology Press, 2013.

[9] Bi Manqing. Electronic technology experiment and curriculum design [M]. 1st edition. Beijing: Machinery Industry Press, 2011: $163 \sim 165$.

[10] Ma Zhongmei, Jie Shunxin, Zhang Kai, etc. C language application programming[M]. Fourth Edition. Beijing: Beijing University of Aeronautics and Astronautics Press, 2007: $41 \sim 42$. 